\title{
Ionization Structure of Heavy Metals Due to Charge Transfer for the Case of Oxygen and Nitrogen
}

\author{
Belay Sitotaw Goshu ${ }^{1,2}$ \\ ${ }^{1}$ Department of Physics, Dire-Dawa University, Dire-Dawa, Ethiopia \\ ${ }^{2}$ Department of Mathematics, Astronomy and Computing Science, Unisa, South Africa
}

Email address:

belaysitotaw@gmail.com

\section{To cite this article:}

Belay Sitotaw Goshu. Ionization Structure of Heavy Metals Due to Charge Transfer for the Case of Oxygen and Nitrogen. International Journal of Astrophysics and Space Science. Vol. 5, No. 2, 2017, pp. 32-40. doi: 10.11648/j.ijass.20170502.12

Received: March 7, 2017; Accepted: March 17, 2017; Published: May 24, 2017

\begin{abstract}
The main aim of this work is to investigate the effect of charge transfer reaction upon gaseous nebula structure, temperature and recombination coefficients of nitrogen and oxygen. We have been used CLOUDY 90 to determine the ionization structure of nitrogen and oxygen. We have used the abundance of heavy elements relative to hydrogen $\mathrm{He}=-1.07, \mathrm{C}$ $=-3.44, \mathrm{~N}=-4.07, \mathrm{O}=-3.31$ and $\mathrm{Ne}=-3.91$. Ionization structure of hydrogen, helium, oxygen and nitrogen, electron temperature and the recombination coefficient are compared with the effective temperatures of $75000 \mathrm{~K}$ and $100000 \mathrm{~K}$ with the luminosity intensity of $10^{38} \mathrm{erg} \mathrm{s}^{-1}$. The result revealed that the ionization structures of elements are highly dependent on the transfer of charge and effective temperatures. In addition, we also tabulate the recombination coefficient of nitrogen and oxygen at different states with temperature of $5000 \mathrm{~K}, 10000 \mathrm{~K}, 15000 \mathrm{~K}$ and $20000 \mathrm{~K}$. This calculation confirms with the results of previous calculation done by different scholars.
\end{abstract}

Keywords: Photoionization, Chemical Abundances, Charge Transfer

\section{Introduction}

In charge reactions, a singly charged ion such as $\mathrm{H}^{+}$or $\mathrm{O}^{+}$ collides with neutral atom or molecule and captures one of its electrons, thereby becoming a neutral atom. Only a small of energy is transferred to the electron donor, so the newly formed neutral retains most of the original energy of the ion. Since it does not longer carry a charge, the neutral atom is not affected by electric and magnetic fields and travels along a straight line of sight path from the point where it was formed.

Different result in the last few decades shows that heavy elements have impact on photoionization model of gaseous nebulae. In particular the major abundant elements like $\mathrm{C}, \mathrm{N}$, and $\mathrm{O}$ play a great role for the formation of planetary nebulae. In this work we focus on the impacts of these elements on assessing the model of photoionization and the ionization structure, temperature of electrons by evaluating the recombination coefficients of nitrogen and oxygen. Moreover, we will investigate the intensity ratio of the spectrum [O I], [O II], [O III] and [N II] in the presence and absence of charge exchange between hydrogen ions and oxygen or nitrogen atoms.

In this paper we argue that the role of charge exchange reaction of $\mathrm{N}$ and $\mathrm{O}$ abundances in modeling gaseous nebula. It is based on the work of [1] on finding nitrogen and oxygen ionization structure, radiative recombination coefficients [2], charge transfer in collisions of $\mathrm{O}^{+}$, with $\mathrm{H}$ and $\mathrm{H}^{+}$with $\mathrm{O}$. This study will focus on the relation of temperature with abundances of heavy elements, the ionization structure and the recombination of each ion at different temperatures and we also compare this work on the work that was done by [3].

In section 2 we present the physics of the charge transfer reaction. In section 3 we present the model of the ionization structure, and describing the model parameters to assess the effect of those parameters with the abundances of $\mathrm{N}$ and $\mathrm{O}$ stated by [4] on the ionization structure and recombination coefficient are then discussed. In section 4 result and discussion presented and conclusion is given in section 5 .

\section{Physics of the Charge Transfer}

Charge transfer is one of a fundamental process in atomic 
physics. It works based on the basic principles of charge conservation. Charge can transfer from one atom or molecule to the other that gets into an excited state, which is possible by releasing an electromagnetic radiation. If the gas is collisionally ionized, the recombination of the ions as well as the ionization balance, is dominated by radiative and dielectronic recombination. However, in photoionized gas the electron density, charge exchange or electron transfer can play an important role in the recombination of the gas.

Charge transfer of the ions of heavy elements with neutral hydrogen is an important process in gaseous nebulae. [5] showed that the charge exchange reaction between $\mathrm{O}^{+}$and $\mathrm{H}^{+}$ could explain observed [O II] line fluxes in planetary nebulae. [6] concluded from an analysis of NGC 7027 that the recombination coefficients of many ions were significantly affected by charge exchange with neutral $\mathrm{H}$ or He. Collisions between singly charged ions and neutral atoms were first discussed by $[7,8]$

Atomic processes are important in determining the ionization equilibrium of particular light elements, especially near the outer boundaries of radiation bounded value nebulae. This process is a charge exchange in two body reactions with hydrogen. We take into account the difficulty to encounter in producing acceptable [O I] [O II], [O III], [N II] and [N III] lines strength in this model. In order to establish the ionization equilibrium of neutral oxygen, hydrogen-ion (atom) charge exchange. The charge transfer equation between hydrogen ion and oxygen with the change of energy released through the processes is given below as

$$
H^{+}+O \rightleftarrows H+O^{+} \Delta E
$$

The importance of the charge transfer reaction is to determine the ionization of oxygen in the interstellar medium. $\Delta E$ is the charge exchange transfer energy which is $0.02 \mathrm{eV}$ [9]. This reaction consider an originally neutral $\mathrm{O}$ atoms into an $\mathrm{O}^{+}$and $\mathrm{H}$, in addition, the ionization potentials of $\mathrm{O}$ and $\mathrm{H}$ are very nearly the same, so that the reaction is very nearly a resonance process. For both of these reasons the cross section for this charge exchange process is relatively large $[9,10]$.

The photoionization cross section used in this work can be calculated from [10]. The photoionization absorption cross sections of these elements are shown in Figures 1 and 2. Since photoionization of heavy metals affected by different factors, it has complex photoionization resonance that leads to dramatic changes in photoionization cross section of the elements. Fig. 2 depicts the photoionization cross section of oxygen atoms when the $h v \geq 13.62 \mathrm{eV}$ and similarly Fig. 1 depict the cross section of hydrogen, helium atoms and respective ions. In both cases, when the ionized energy is greater than threshold energy of elements, the photoionization cross section diminished rapidly with energy.

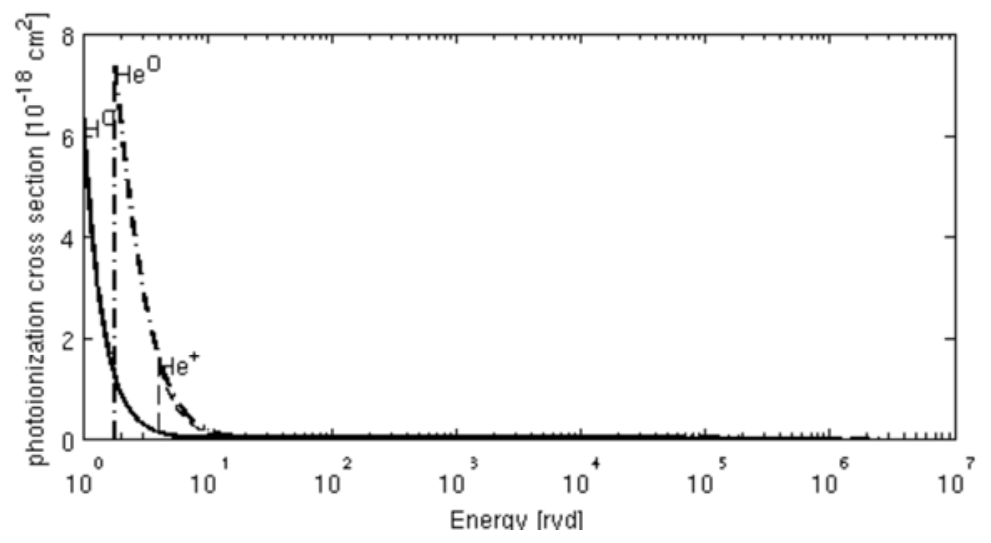

Figure 1. Photoionization cross section of $\mathrm{H}, \mathrm{He}, \mathrm{He}^{+}$.

The cross sections of oxygen and nitrogen atoms were determined by using the quantum defect method as formulated by [11] and fitted using interpolation formula [12]. It is highly dependent on the ionizing energy.

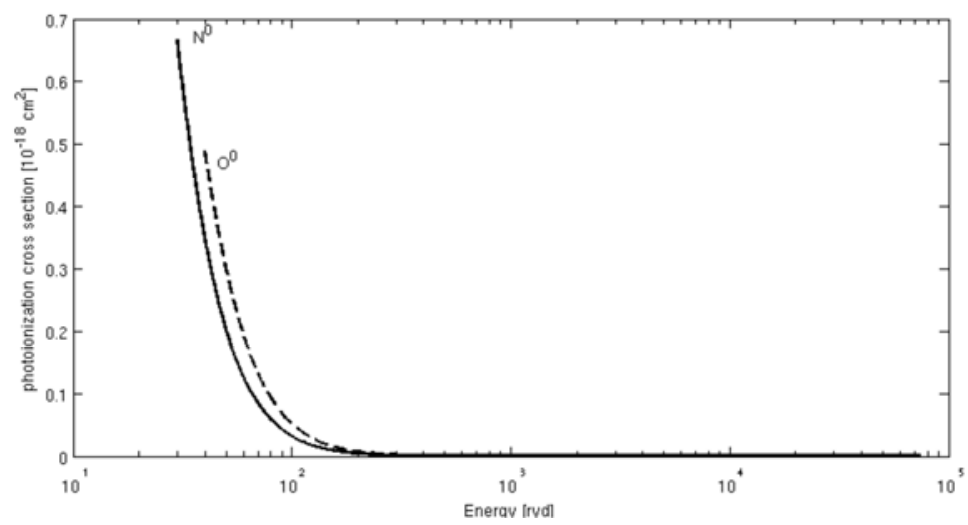

Figure 2. Photoionization of oxygen atom and ions. 
Metal ions have many different thresholds for photoionization, depending on which of many electrons present to be kicked out of the atom. This is the result in the ionization cross section having "edges" [10]. Neutral oxygen atom $\mathrm{O}^{+}$can be ionized by when ionization potential is greater than $13.62 \mathrm{eV}$. The ionization potential of $\mathrm{H}, \mathrm{He}$, $\mathrm{He}^{+}, \mathrm{N}$ and $\mathrm{O}$ are shown below at different levels shown below.

$\begin{array}{ll}\text { Element } & \text { Ionization Potential } \\ \mathrm{H} \rightarrow \mathrm{H}^{+} & h v=13.6 \mathrm{eV} \\ \mathrm{He} \rightarrow \mathrm{He}^{+} & h v_{h e}=24.6 \mathrm{eV} \\ \mathrm{N} \rightarrow \mathrm{N}^{+} & h v_{N}=14.53 \mathrm{eV} \\ \mathrm{O} \rightarrow \mathrm{O}^{+} & h v_{O}=13.62 \mathrm{eV}\end{array}$

A work which was done by [13] has also shown the ionization of nitrogen is influenced by the reaction

$$
H^{+}+N \leftrightarrows H+N^{+}+\Delta E
$$

When photoionization rate is low as would occur near the ionization edge of a nebula. In this limit reaction rates for change exchange exceed those of photoionization and electron recombination; at the electron temperatures encountered in planetary nebula they tend to condition that

$$
\frac{N^{+}}{N} \approx \frac{O^{+}}{O} \approx \frac{H^{+}}{H}
$$

That is, charge transfer produced similar levels of ionization for $\mathrm{N}, \mathrm{O}$ and $\mathrm{H}$. Following this relations given by Eq.3, can be rewrite by $[8,13$ and 14$]$ as

$$
\frac{N\left(O^{+}\right)}{N(O)}=\frac{8}{9} \frac{N\left(H^{+}\right)}{N(H)}
$$

The ionization potential of $\mathrm{N}(14.53 \mathrm{eV})$ is also close to that of $\mathrm{H}(13.60 \mathrm{eV})$, but the ionization potential of $\mathrm{O}$ is less than $\mathrm{N}$. On comparing $\mathrm{N}$ and $\mathrm{O}$, we see that oxygen has a fourth electron in its $2 p$ subshell. Since this subshell contains only three orbitals, this fourth electron must be paired in $2 p$ subshell.

Charge exchange between oxygen atom and hydrogen ion is the most rapid gas-phase process affecting proton in atomic gas is not recombined with electron but charge exchange with neutral oxygen. It was first described by [8].

$$
N\left(X^{0}\right)\left[\int_{0}^{\infty} 4 \pi J^{v} a_{v}\left(X^{0}+N\left(H^{+}\right) d v \beta\right]=N\left(X^{+}\right)\left[N_{e} \alpha\left(X^{0}+N\left(H^{0}\right)\right] \beta^{\prime}\right.\right.
$$

Where $\mathrm{X}^{0}$ and $\mathrm{X}^{+}$represent $\mathrm{O}^{0}, \mathrm{~N}^{0}$ and $\mathrm{O}^{+}, \mathrm{N}^{+}$, respectively, the charge exchange rate coefficients $\beta$ and $\beta$,' and $\alpha\left(\mathrm{X}^{0}\right)$ is the recombination coefficient of atom and $a_{v}$ is the photoionization cross section and $N_{e}$ is the number of electron per unit volume.

The rate of coefficients in the forward time reaction per unit volume per unit time for the reaction can be written

$$
N\left(U^{0}\right) N_{e} \beta
$$

Where $\beta$ is expressed in terms of the reaction cross section
The transfer processes between any atom and hydrogen are important at the edge of hydrogen ionization. This effect is important for prediction of emission lines [N I] and [O I] since collisional excitation to occurs, it is necessary atoms exists in $\mathrm{H}$ II regions.

The reaction $\mathrm{H}^{+}+\mathrm{O} \leftrightarrows \mathrm{H}+\mathrm{O}^{+}$is important in driving interstellar chemistry because it transfers ionization to oxygen which can then readily participate in chemistry. Because the ionization potentials are close, and has a large statistical weight relative to nitrogen and oxygen.

Charge exchange was once believed to be important rate in the ionization balance of $\mathrm{N}$ in the outer boundaries of ionization bounded nebulae. One of the characterizations of photoionization of a gas by an external source of radiation is that elements nitrogen and oxygen remain ionized to a distance somewhat beyond the point where hydrogen becomes neutral, in spite of the fact, the photoionization of $\mathrm{N}$ and $\mathrm{O}$ are greater than that of $\mathrm{H}$. This is due to the fact that the photoionization cross sections of $\mathrm{N}$ and $\mathrm{O}$ large at high frequencies, relative to their threshold values, than that of $\mathrm{H}$.

As result of this near ionization edge of the gas $\mathrm{N}$ and $\mathrm{O}$ can absorb radiation has not yet produced high optical depth. Furthermore, because the ionization edge of nebulae is caused primary by absorption, it is possible to have large radiation densities at frequencies far removed from the Lyman continuum which are capable of producing high ionization. Thus, in a nebula with a very hot central star, one can find that a high degree of ionization for $\mathrm{N}$ and $\mathrm{O}$, ie. $\mathrm{N}^{+3}$ and $\mathrm{O}^{+2}$ are right up to the point where $\mathrm{H}$ becomes neutral.

\section{Model}

This model is applied in HII regions to study the ionization structure and recombination coefficients of nitrogen and oxygen. We have devoted on the solution of the ionization equilibrium of gas, including heavy metals like $\mathrm{H}, \mathrm{He}, \mathrm{C}, \mathrm{N}$, $\mathrm{O}$ and $\mathrm{Ne}$. The technique used in this work is simulation using CLOUDY C10.00 [16] and the solar abundances developed by [4]. In order to determine the effect of charge exchange on the ionization, we will test other model computed by [17]. The ionization of oxygen and nitrogen is written as

$\sigma(v)$ and given by

$$
\beta(T)=\int_{0}^{\infty} v \sigma(v) f(v) d v
$$

Where $f(v)$ is the Maxwell-Boltzmann distribution functions for the relative velocity $v$ in the $\mathrm{OH}$ center-ofmass system, and thus involves their reduced mass. Likewise the rate for the inverse relation can be written

$$
N\left(O^{+}\right) N_{e} \beta^{\prime}(T)
$$

Further studied of ionized nebulae included [18], who 
noted that because of charge transfer the ionization of oxygen should be coupled to that of hydrogen on most astrophysical situations, according to

$$
\beta(T)=\frac{8}{9} \beta^{\prime}(T)
$$

It is applicable for $T \geq 10^{4} \mathrm{~K}$ and $\mathrm{KT} \gg \Delta E$. As a result of this oxygen does not ionized in the outer parts of nebulae, as do other heavy elements.

The ionization parameters chosen for this model are:

We use the solar abundances of elements relative to hydrogen, developed by [4]. Out of 30 elements, we only use helium, carbon, nitrogen, oxygen and neon abundances relative to hydrogen in logarithmic

Table 1. Relative abundances of heavy elements with solar composition [4] all values are given as the logarithm number relative to hydrogen.

\begin{tabular}{ll}
\hline Element & Relative abundances \\
\hline $\mathrm{He}$ & -1.07 \\
$\mathrm{C}$ & -3.44 \\
$\mathrm{~N}$ & -4.07 \\
$\mathrm{O}$ & -3.31 \\
$\mathrm{Ne}$ & -3.91 \\
\hline
\end{tabular}

a The luminosity of the star is $1 \times 10^{38} \mathrm{erg} \mathrm{s}^{-1}$.

b We select two central star temperatures (blackbody) as 75000 and $100000 \mathrm{~K}$.

c The transfer of the diffuse ionizing radiation is treated in the outward only,

d Presence of dust negligible,

e The inner radius of the nebula is $0.0102 \mathrm{pc}$,

$\mathrm{f}$ The outer radius of the nebula is free,

$\mathrm{g}$ We select two different gases density per volume of hydrogen are $1 \times 10^{4} \mathrm{~cm}^{-3}$ and $3.1623 \times 10^{4} \mathrm{~cm}^{-3}$.

In addition, we use photoionization CLOUDY codes to take into account charge exchange reactions involving hydrogen and relevant heavy elements like oxygen and nitrogen. The reaction rates are taken from [19] at temperature of $10^{4} \mathrm{~K}$.

The ionization structure of $\mathrm{O}$ and $\mathrm{N}$ are computed in the on-the-spot approximation according to the formulation of [10] which, is the most complete up to now presented, because it considered the $\mathrm{He}^{+}$recombination to the ground level of $\mathrm{He}$ as ionizing source of $\mathrm{H}$ and $\mathrm{He}$ as well as the recombination to the excited levels of $\mathrm{He}$ as ionizing source of $\mathrm{H}$.

\section{Result and Discussion}

We have computed the ionization structure of $\mathrm{N}$, and $\mathrm{O}$ for spherical nebulae with uniform hydrogen density with two effective temperatures 75000 and $100000 \mathrm{~K}$. This model was calculated for each element in order to produce as closely as possible the physical condition stated on [4]. The model developed by three most important elements only. It is validated with other paper done under similar conditions. Our goal is to find the ionization structure of nitrogen and oxygen under charge exchange/transfer reactions based on the assumption described in section 3.2.

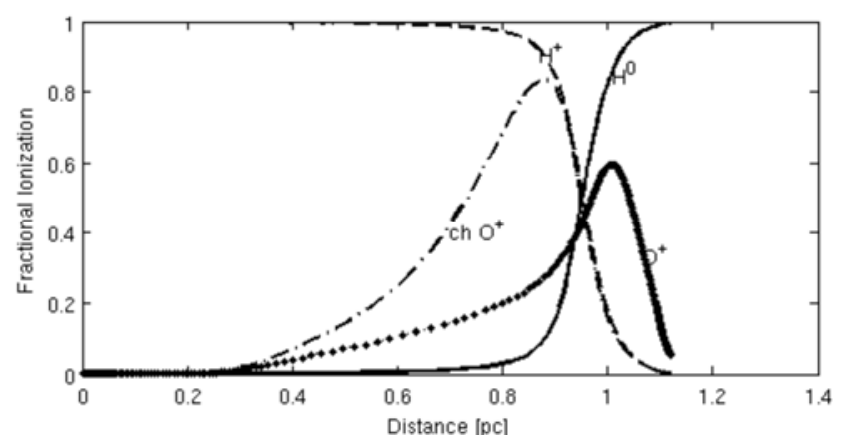

Figure 3. The ionization structure of hydrogen ion and oxygen atom versus with the distance from the central star at $75000 k$ and density per unit volume is $N(H)=10,000 \mathrm{~cm}^{-3}$. The solid line shows neutral hydrogen, broken line with dot shows hydrogen ions, ch $\mathrm{O}^{+}$shows oxygen ion in the presence of charge transfer and only dot shows oxygen ion in the absence of charge transfer.

The model calculation shown in Figures 3-6 depict the ionization structure of elements on the transfer of charge described by $\mathrm{H}^{+}+\mathrm{O} \rightleftharpoons \mathrm{H}+\mathrm{O}^{+}$and $\mathrm{H}^{+}+\mathrm{N} \rightleftharpoons \mathrm{H}+\mathrm{N}^{+}$as it described on this model based on the parameter shown in section 3.1. Moreover; we also assumed that the photoionization of heavy elements was done due to stellar radiation. The ionization structure shown in Fig. 3 only considers photoionization and recombination processes of oxygen and hydrogen based on charge exchange transfer. Recently different scholars have identified the importance of charge transfer in determining the ionization structure of heavy elements [19]. Therefore, the ionization structure shows oxygen doubly ionized in a HII while nitrogen is triply ionized as it shown in Fig. 6.

The abundances ratio of $\mathrm{O}^{+}$and $\mathrm{N}^{+}$are shown in Figures 34 respectively. It increases outward reaching an order of magnitude $0.9 \mathrm{pc}$ in the charge exchange transfer process whereas, in the absence of charge transfer, they attain maximum at about $1.05 \mathrm{pc}$. The charge transfer process is the dominant at which the radius of hydrogen ions, coincide in short time interval than no charge transfer process in both oxygen and nitrogen. This is due to ionization potential energy and statistical weights of the element.

Figure 5 depicts the same nature as the previous result. The abundance of $\mathrm{O}^{+}$when $\mathrm{N}(\mathrm{H})=3.1623 \times 10^{4} \mathrm{~cm}^{-3}$ and temperature $100000 \mathrm{~K}$, it increases outward and reaches a magnitude of $0.62 \mathrm{pc}$ in the charge exchange transfer processes whereas, in the absence of charge transfer processes, it attains peak value at about $0.68 \mathrm{pc}$ by increasing outward.

Figure 6 depicts The abundances of $\mathrm{N}^{+}$at $\mathrm{N}(\mathrm{H})=3.1623 \times$ $10^{4} \mathrm{~cm}^{-3}$ and temperature $100000 \mathrm{~K}$. Its radius increases outward and reaches peak values at $1.38 \mathrm{pc}$ in the charge exchange process whereas, in the absence of charge transfer processes, its radius reaches peak value at about $1.52 \mathrm{pc}$.

The comparison of the present works with [8] shows an agreement in general. However, there are some significant differences are found in the solar abundances of element and temperature of blackbody. Due to this, there is a variation in peak value at which the reaction attains equilibrium. 


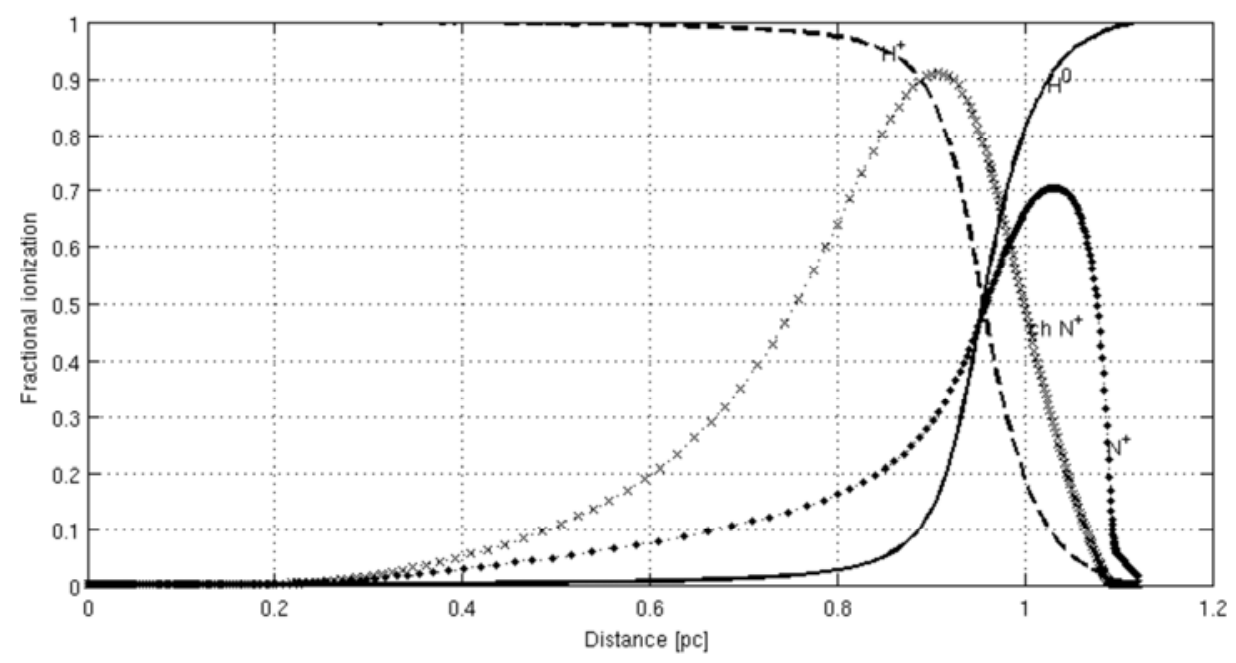

Figure 4. The ionization structure of nitrogen atom relative to hydrogen with hydrogen ions at $75000 \mathrm{k}$ and density per unit volume is $N(\mathrm{H})=10000 \mathrm{~cm}^{-3}$ versus with the distance from the central star in case of charge transfer and no charge transfer. Black solid dot represent nitrogen ions in the absence of charge transfer whereas, cross shows the nitrogen ions in the presence of charge transfer (ch), solid line shows neutral hydrogen and broken line shows hydrogen ions.

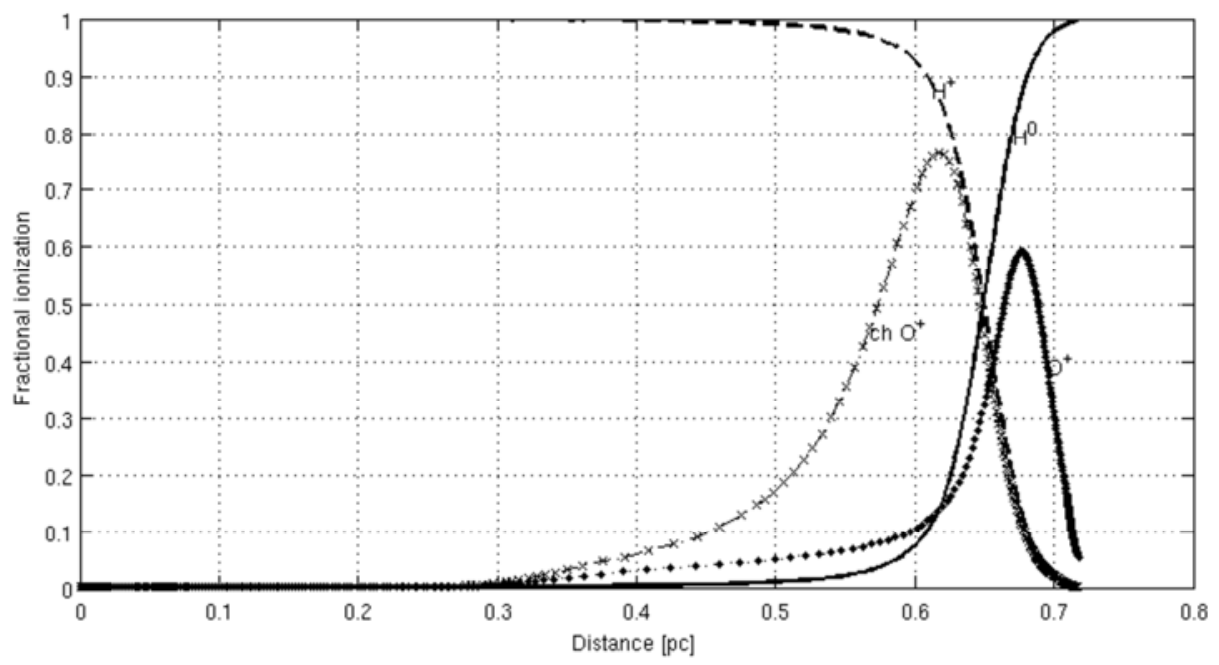

Figure 5. The ionization structure of oxygen atom relative to hydrogen at $100000 \mathrm{~K}$ and density per unit volume is $N(H)=3.1623 \times 10^{4} \mathrm{~cm}^{-3}$ versus with the distance from the central star.

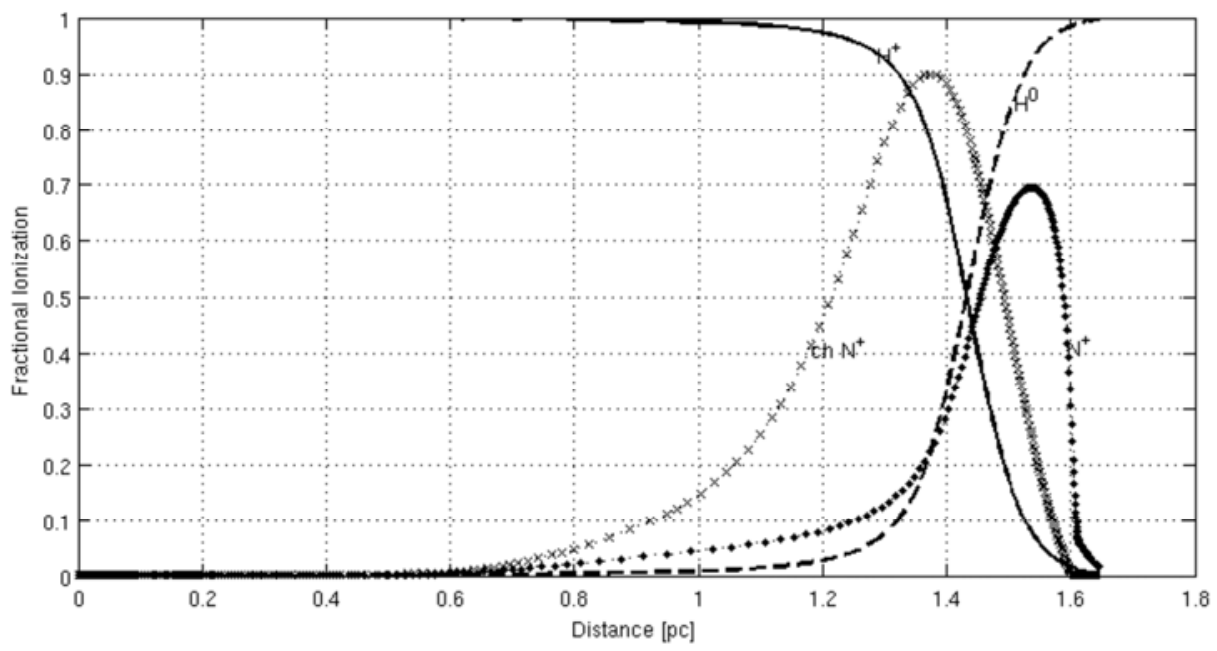

Figure 6. Depicts the ionization structure of nitrogen atom relative to hydrogen at $100000 \mathrm{k}$ and density per unit volume is $N(\mathrm{H})=3.1623 \times 10^{4} \mathrm{~cm}^{-3}$ versus with the distance from the central star. 
The nebula has a density of $1 \times 10^{4} \mathrm{~cm}^{-3}$, a filling factor 0.005 , and ionized by $75000 \mathrm{~K}$ blackbody with total luminosity intensity $\mathrm{L}=10^{38} \mathrm{erg} \mathrm{s}^{-1}$ and figure 9 shows ionization structure of hydrogen and nitrogen at different stages as a function of radius. The charge transfer rate is 9.48 $\times 10^{-13} \mathrm{~cm}^{3} \mathrm{~s}^{-1}[17]$.

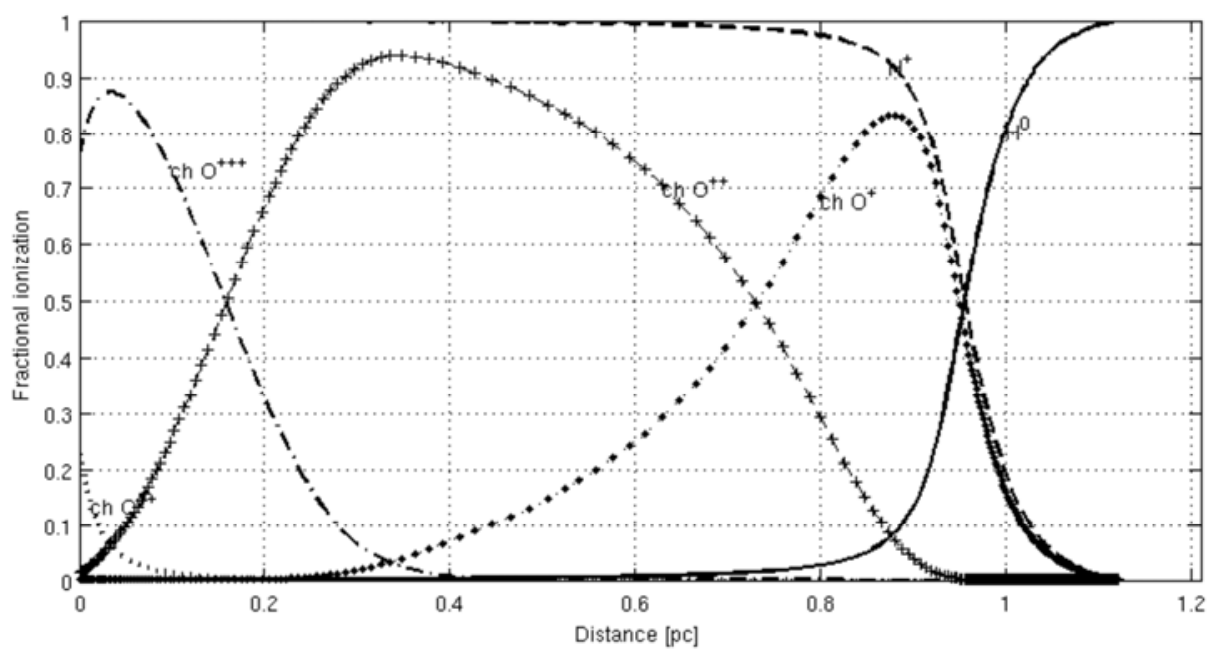

Figure 7. Model Calculations: the ionization structure of typical planetary nebulae of oxygen abundances at different stages.

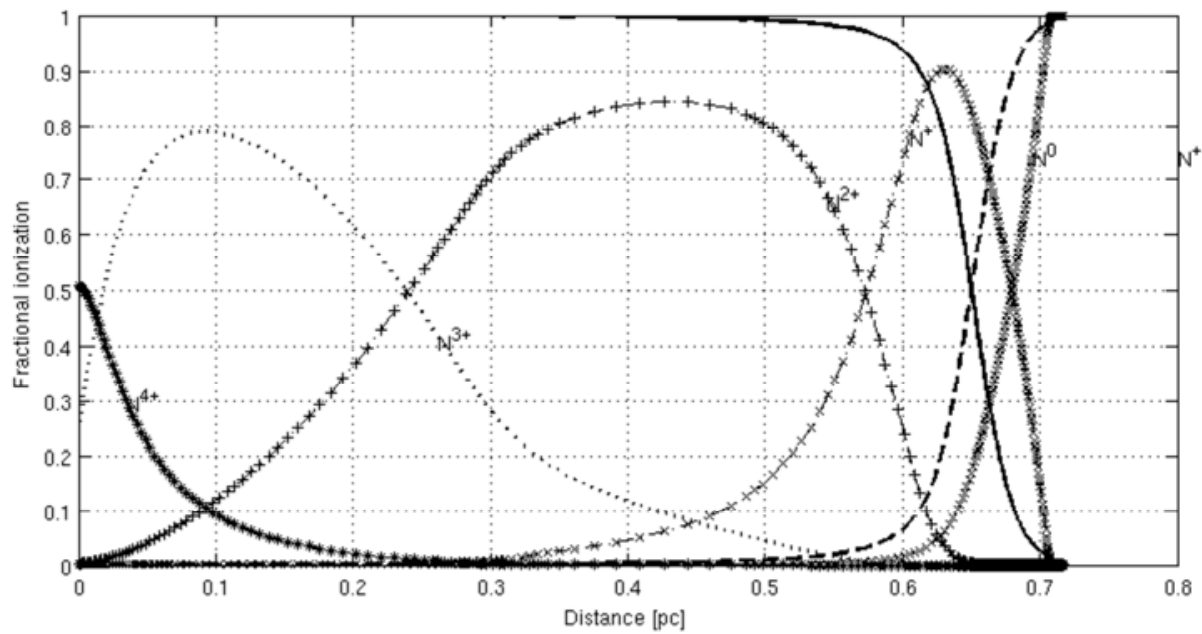

Figure 8. Model Calculation: the ionization structure of typical planetary nebulae of nitrogen abundances at different stages. The nebula has a density of $3.1623 \times 10^{4} \mathrm{~cm}^{-3}$, a filling factor 0.005 , and ionized by $1 \times 10^{5} \mathrm{~K}$ blackbody with total luminosity intensity $L=1 \times 10^{38}$ erg $\mathrm{s}^{-1}$. The figure shows hydrogen and nitrogen ions fraction as a function of radius. The charge transfer rate is $\$ 5.3 \times 10^{-10} \mathrm{~cm}^{3} \mathrm{~s}^{-1}$.

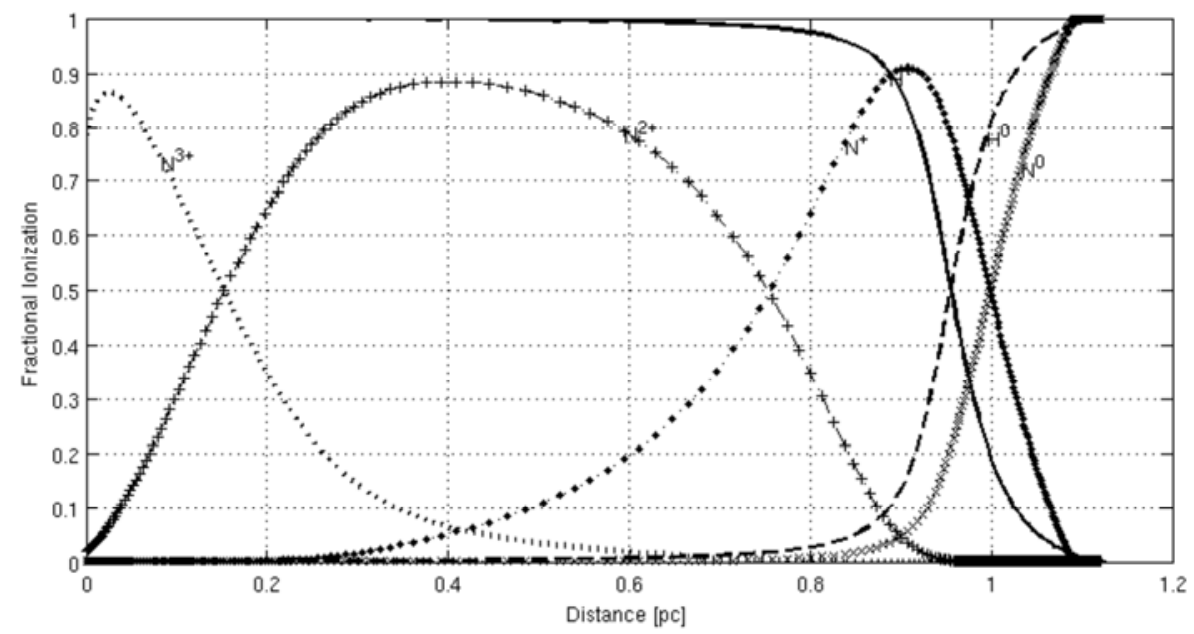

Figure 9. Model Calculation: This figure shows the ionization structure of a of nitrogen at different stages. 


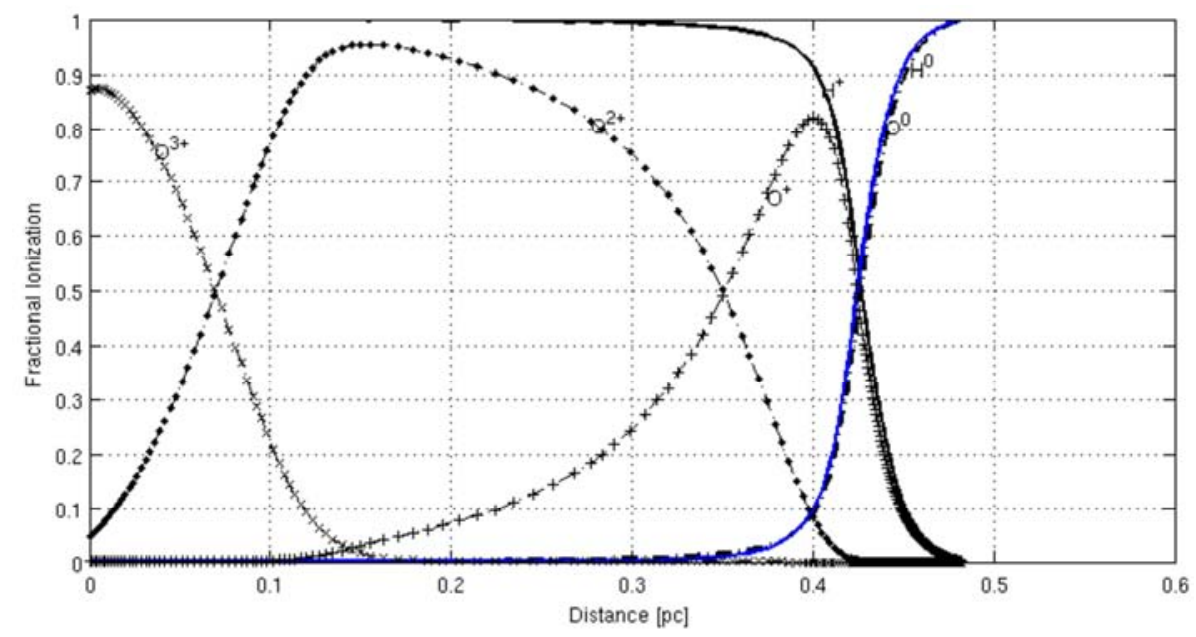

Figure 10. Model Calculation: This figure shows the ionization structure of typical planetary nebulae of oxygen abundances at different stages ionizations. The nebula has a density of $3.1623 \times 10^{4} \mathrm{~cm}^{-3}$, a filling factor 0.005 , and ionized by $75,000 \mathrm{~K}$ blackbody with total luminosity intensity $L=1 \times 10^{38}$ erg $\mathrm{s}^{-1}$. The figure shows hydrogen fraction and nitrogen ions as a function of radius. The charge transfer rate is $5.9 \times 10^{-10} \mathrm{~cm}^{3} \mathrm{~s}^{-1}$.

Figures 7 and 10 depict models calculation of oxygen and hydrogen at different stages of ionization at temperature of $75,000 \mathrm{~K}$, hydrogen number density 10,000 and $3.1623 \times 10^{4}$ $\mathrm{cm}^{-3}$. The nature of ionization structure both ions are almost the same but the distance at which they attains and to be neutral is quite different. For the low density, it extends up to $1.1 \mathrm{pc}$ whereas, for high density, it is almost neutral before
$0.5 \mathrm{pc}$. This is due to the concentration of number density of hydrogen and its abundances. The ionization structure of oxygen and nitrogen shown in Figures 7 and $9, \mathrm{O}^{++}$and $\mathrm{N}^{++}$ are present in respective figures in almost equal amounts. Moreover, as $\mathrm{O}^{+}$and $\mathrm{N}^{+}$are increase to attain maximum values, $\mathrm{O}^{++}$and $\mathrm{N}^{++}$disappear. Since $\mathrm{O}^{+}$and $\mathrm{N}^{+}$are tied to ionization of hydrogen through charge exchange.

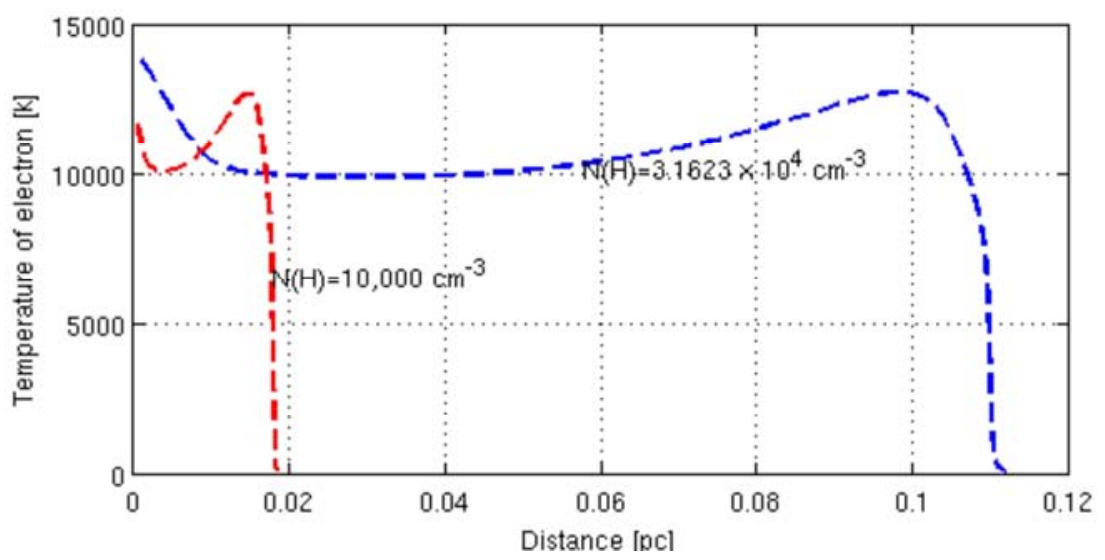

Figure 11. Variation of electron temperature in planetary nebulae which is optically thick in the Lyman continuum and has black body central temperature 100,000 K computed using the heavy elements abundances of [4].

The result of the numerical solution of the thermal balance equation, taking into account all heating and cooling processes are shown in Figures.11 and 12 for central temperatures $1 \times 10^{5}$ and $7.5 \times 10^{4} \mathrm{~K}$ respectively, different hydrogen density. The figures clearly show that there are variations of electron temperature. We have also seen from figures the nature of temperature depends with hydrogen number densities. At hydrogen density of $10000 \mathrm{~cm}^{-3}$, the result shows the temperature of electron decrease slowly until the distance from the central star is about $0.07 \mathrm{pc}$ and beyond this the temperature is almost uniform. When the distance is beyond $0.1 \mathrm{pc}$, it decreases sharply. At hydrogen density $3.1263 \times 10^{4} \mathrm{~cm}^{-3}$ the nature of the temperature structure is almost the same as [23] and it changes slowly with the distance.

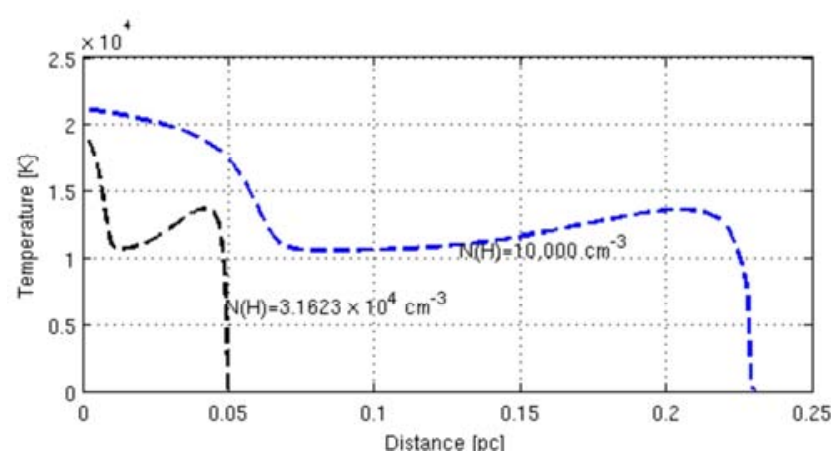

Figure 12. The Variation of electron temperature in planetary nebulae which is optically thick in the Lyman continuum and has black body central temperature $75000 \mathrm{~K}$ computed using the heavy elements abundances of [4]. 
Table 2. Radiative recombination coefficients of nitrogen and its ions at different temperatures based on the present model in the order of $10^{-13} \mathrm{~cm}^{3} \mathrm{~s}^{-1}$ at effective temperature of $75000 \mathrm{~K}$.

\begin{tabular}{llllll}
\hline Temperature & $\mathbf{N}$ & $\mathbf{N}^{+}$ & $\mathbf{N}^{+2}$ & $\mathbf{N}^{+3}$ & $\mathbf{N}^{+4}$ \\
\hline $5000 \mathrm{~K}$ & 5.99 & 37.4 & 90.4 & 153.0 & 237.0 \\
$10000 \mathrm{~K}$ & 3.76 & 24.0 & 96.3 & 148.0 & 253.0 \\
$15000 \mathrm{~K}$ & 2.89 & 18.4 & 44.2 & 150.0 & 195.0 \\
$20000 \mathrm{~K}$ & 2.45 & 15.2 & 36.5 & 60.0 & 91.0 \\
\hline
\end{tabular}

Table 3. Radiative recombination coefficients of oxygen ions at different temperatures based on the present model in the order of $10^{-13} \mathrm{~cm}^{3} \mathrm{~s}^{-1}$.

\begin{tabular}{lllll}
\hline Temperature & $\mathbf{O}$ & $\mathbf{O}^{+}$ & $\mathbf{O}^{\mathbf{+ 2}}$ & $\mathbf{O}^{+\mathbf{3}}$ \\
\hline $5000 \mathrm{~K}$ & 4.48 & 33.4 & 89.7 & 165.0 \\
$10000 \mathrm{~K}$ & 2.72 & 21.3 & 57.5 & 106.0 \\
$15000 \mathrm{~K}$ & 2.03 & 16.3 & 81.2 & 120.0 \\
$20000 \mathrm{~K}$ & 1.67 & 13.5 & 136.5 & 67.2 \\
\hline
\end{tabular}

For comparative analysis the result we obtained in this work are tabulated in Table 2 and 3 with the result derived by [10] was given in Table 4. As we can see from Tables 2 and 3 the radiative recombination of nitrogen is greater than oxygen in all cases. The result shows that the radiative recombination of nitrogen is highly dependent on temperature. Moreover, at low temperature $T_{e}<10000 \mathrm{~K}$, the result we obtained and the previous work done by [22] agree for radiative coefficients of nitrogen.

Table 4 summarizes our best estimates of radiative recombination coefficients of nitrogen and oxygen at different stages of ionization which are compared with [10, 17, 3, and 22] and the present models. In the present model we consider the thermal balance to explain the recombination coefficients of ions. The recombination coefficients calculated by different authors are somewhat different from this model. This is due to different values of charge exchange coefficients and abundances of elements.

Table 5 shows that [O $\mathrm{II}]$ intensity ratio tends to be slightly greater than by $10 \%$ when charge transfer takes place. According to our discussion in section 2, charge transfer $\mathrm{H}+\mathrm{O}^{+} \rightleftharpoons \mathrm{H}^{+}+\mathrm{O}$ is the dominant processes and the abundances of $\mathrm{O}^{+} / \mathrm{H}^{+}$is less relative to the other higher ions of oxygen in this region. This can increase the ratio of intensity at large values. The same is true for [O II] ratios. The lines ration increased more than $10 \%$, whereas, [O III] the charge transfer is described as $\mathrm{H}+\mathrm{O}^{++} \rightleftharpoons \mathrm{H}^{+}+\mathrm{O}^{+}$. The result shows that the ratio of lines when this reaction takes places less than in the absence of charge transfer. The lines ratio changes by more than $50 \%$ when the charge transfer takes places. Research done [21] and this new results show that $[\mathrm{O}$ III] $\lambda 5007 A$ lines 100 times more intense than the aurora line [O III] $\lambda 4363 A$, and can be seen clearly. This is because of higher oxygen abundances, optical lines act as the main coolant for cooling and lowering the electron temperatures.

As we can see from Table 5 for emission lines of nitrogen is increased by large values when charge transfer takes places. Charge transfers processes hardly affect the total amount of [N II] ratio of emission lines. Since the charge transfer of nitrogen is described by $\mathrm{H}+\mathrm{N}^{+} \rightleftharpoons \mathrm{H}^{+}+\mathrm{N}$ depend on the chemical abundances of ions and temperature.
Table 4. Comparison of Radiative recombination coefficients of oxygen and nitrogen ions with the previous results in the order of $10^{-13}\left[\mathrm{cms}^{-1} \mathrm{~s}^{-1}\right.$ at temperature $10000 \mathrm{~K}$.

\begin{tabular}{lllll}
\hline $\mathbf{X}^{+\mathbf{i}}$ & $\mathbf{1}$ & $\mathbf{2}$ & $\mathbf{3}$ & $\mathbf{4}$ \\
\hline $\mathrm{N}^{0}$ & 3.92 & 3.14 & 4.1 & 3.76 \\
$\mathrm{~N}^{+}$ & 22.8 & 22.0 & 22.0 & 24.0 \\
$\mathrm{~N}^{+2}$ & 54.4 & 52.4 & 50.0 & 96.3 \\
$\mathrm{~N}^{+3}$ & 95.5 & 86.6 & 65.0 & 148.0 \\
$\mathrm{O}^{0}$ & 3.31 & & & 2.72 \\
$\mathrm{O}^{+}$ & 20.5 & & & 21.3 \\
$\mathrm{O}^{+2}$ & 54.3 & & & 57.5 \\
$\mathrm{O}^{+3}$ & 103.0 & & & 106.0 \\
\hline
\end{tabular}

$1=[1]$

$2=[15]$

$3=[3]$

$3=[3]$

$4=$ New Model

Table 5. Line fluxes of oxygen and nitrogen relative to line flux of $H \beta$ at temperature $10000 \mathrm{~K}$ when $\mathrm{H} \beta=1.0$.

\begin{tabular}{llll}
\hline & & No Charge transfer & Charge transfer \\
\hline Ions & $\boldsymbol{\lambda}[\boldsymbol{A}]$ & Ratio of lines & \\
\hline$[\mathrm{O} \mathrm{I}]$ & 6300 & 0.060 & 0.595 \\
& 6363 & 0.019 & 0.190 \\
{$[$ O II] } & 3727 & 1.941 & 4.083 \\
& 3729 & 0.637 & 1.285 \\
& 3726 & 1.295 & 2.785 \\
& 7323 & 0.133 & 0.350 \\
& 7332 & 0.108 & 0.283 \\
O 3 & 1661 & 0.021 & 0.009 \\
& 1666 & 0.05 & 0.023 \\
& 5007 & 10.933 & 4.842 \\
& 4959 & 3.632 & 1.609 \\
& 4363 & 0.075 & 0.034 \\
& 6584 & 0.907 & 2.391 \\
& 6548 & 0.307 & 9,810 \\
& 5755 & 0.017 & 0.052 \\
\hline
\end{tabular}

\section{Conclusions}

Using the CLOUDY photoionization code, we were able to calculate the ionization structure of nitrogen and oxygen on charge transfer reaction has substantial affected by blackbody temperature. When the temperature of the blackbody is 75000 and $100000 \mathrm{~K}$, the oxygen atom rapidly reacted with hydrogen ions in both cases whereas, nitrogen are slightly slow relative to oxygen. We attribute the phenomena of charge transfer on oxygen and nitrogen to study the ionization structure and line ratio. We have tested the charge transfer reaction at different hydrogen number densities per unit volume. It also show that the transfer of charge through interaction of hydrogen ions more rapid than the charge transfer of hydrogen ion with neutral of nitrogen. The ratio of lines depends on charge transfer between ions and atoms. [O I] and [O II] ratios increased by more than $10 \%$ when charge transfer takes place. Whereas [O III] ratios decreased almost by half when charge transfer takes places. Similarly, the ratio of lines [N II] increased by more than $10 \%$ when charge transfer takes places. This is because of the abundances of ions in those particular distances.

We have checked the radiative recombination coefficients 
of oxygen and nitrogen ions. There is some discrepancy of results with the previous results done by [10, 17, 3, and 22]. We can enhance the radiative recombination coefficients and intensity rations of ions by changing the abundances of elements and effective temperature of the source.

\section{References}

[1] Wilkes B. J., Ferland G. J., Hanes D. and Truran J. W. (1981). Cloudy 90 Numerical Simulations of Plasma and their Spectra, NMRAS 197-1w.

[2] Peimbert M., 1975, Ann. Rev. Astron. Astrophys., 13, 113.

[3] Aldrovandi S. M. V and Pe'quignot D. (1973). Radiative and Dielectronic Recombination coefficients for complex ions, Astro. Astrophys, 25, 137.

[4] Asplund E., Grevesse N., Sauval A., and Scott P. (2009). The Chemical Composition of the Sun, 2009, ARAA, 47, 481.

[5] Williams R. E. (1973). Mon. NOt R. astr. Soc., 164, 111-119.

[6] Perquignot, D. Aldrovandi, S. M. V. and Stasinska, G. (1978). Charge Transfer Reaction: A consistent Model of the Planetary Nebulae NGC 7027, A\&A, 63, 313.

[7] Chamberlian J. W., 1956, ApJ, 124, 390.

[8] Field G. \& Steigman G. (1971). Charge transfer and ionization equilibrium in the interstellar medium, Astrophysics journal, $166,59$.

[9] Steigman G. (1974). Charge transfer reactions in multiply charged ion-atom collisions. ApJ, 199, 642-646.

[10] Osterbrock E. D. (1989). Astrophysics of Gaseous Nebulae and Active Galactic Nuclei, California University of Science Book.
[11] Peach G. (1967). MeM. R. A. S., 71, 13.

[12] Seaton M. J., (1969). The ionization structure of planetary nebulae-VII. The heavy elements. MNRAS, 146, 171F.

[13] Watson W. D., Gas Phase Reactions in Astrophysics, 1978, ARA\&A, 16: 585-615.

[14] Brown R. L., 1972, Ap. and Space. Sci., 16, 274.

[15] Mallik D. C. V. (1975). Temperature and Emission line Structure at the Edges of HII regions, ApJ, 197, 355-363.

[16] Ferland G. J. et al., 2010, Hazy a brief introduction to CLOUDY C10.00.

[17] Perinotto M. (1977). On the Nitrogen and Oxygen Abundances in Nebulae, A \& A, 61, 247-249.

[18] Davidson K. and Tucker W., 1970, ApJ, 161, 437.

[19] Peimbert M., Luis F. Rodriguez and Siliva, Torres-Peimbert (1974). Ionization Structure of Gaseous Nebulae: Sulphur, Nitrogen and Helium, Nacional Autonoma de Mexico, Vol 1.

[20] Aldrovandi S. M. V and Pe'quignot D., 1976, Astro. Astrophys, 25, 137-140.

[21] Castellanos M., Dia'z A. I., and Terlevich E. (2002). A Comprehensive study of reported high giant H II regions- I. Detailed abundances analysis. Mon. Not. R. Astron. Soc. 329, 315.

[22] Fang X., Storey P. J., and Liu X. W. (2011). New effective recombination coefficients for nebular N II lines. A\&A, 530, A18.

[23] Flower D. R and Seaton M. J. (1969). The Ionization Structure of Planetary Nebulae: V III the Heavy Elements, MNRAS, $146,243-263$. 